\title{
MicroRNA-146a inhibition promotes total neurite outgrowth and suppresses cell apoptosis, inflammation, and STAT1/MYC pathway in PC12 and cortical neuron cellular Alzheimer's disease models
}

\author{
Yinghui $\mathrm{Ma}^{1 * \bullet}$, Jiye $\mathrm{Ye}^{1 * \oplus}$, Li Zhao ${ }^{1 \oplus \bowtie}$, and Dongmei $\mathrm{Pan}^{2 \oplus \varpi}$
}

${ }^{1}$ Department of Neurosurgery, Huangshi Central Hospital, Affiliated Hospital of Hubei Polytechnic University, Huangshi, China ${ }^{2}$ Department of Gerontology, Huangshi Central Hospital, Affiliated Hospital of Hubei Polytechnic University, Huangshi, China

\begin{abstract}
This study aimed to explore the effect of microRNA (miR)-146a inhibition on regulating cell apoptosis, total neurite outgrowth, inflammation, and STAT1/MYC pathway in Alzheimer's disease (AD). PC12 and cortical neuron cellular AD models were constructed by A $\beta 1-42$ insult. For the former model, nerve growth factor (NGF) stimulation was previously conducted. miR-146a inhibitor and negative-control (NC) inhibitor were transfected into the two cellular AD models, and then cells were named miRinhibitor group and NC-inhibitor group, respectively. After transfection, cell apoptosis, total neurite outgrowth, supernatant inflammation cytokines, and STAT1/MYC pathway were detected. miR-146a expression was similar between PC12 cellular AD model and control cells (NGF-stimulated PC12 cells), while miR-146a expression was increased in cortical neuron cellular AD model compared with control cells (rat embryo primary cortical neurons). In both PC12 and cortical neuron cellular AD models, miR-146a expression was reduced in miR-inhibitor group compared with NC-inhibitor group after transfection. Furthermore, cell apoptosis was attenuated, while total neurite outgrowth was elevated in miR-inhibitor group compared with NC-inhibitor group. As for supernatant inflammatory cytokines, tumor necrosis factor- $\alpha$, interleukin (IL)-1 $\beta$, IL-6, and IL-17 levels were lower in miRinhibitor group than in NC-inhibitor group. Additionally, STAT1 and c-Myc mRNA and protein expressions were attenuated in miR-inhibitor group compared with NC-inhibitor group. In conclusion, miR-146a potentially represented a viable therapeutic target for $\mathrm{AD}$.
\end{abstract}

Key words: Alzheimer's disease; MicroRNA 146a; STAT1/MYC; Total neurite outgrowth; Cell apoptosis; Inflammation

\section{Introduction}

Alzheimer's disease (AD), the most prevalent neurodegenerative disorder of the elderly population, is a major global public health burden with over 26 million affected $(1,2)$. AD is neuropathologically marked by deposition of abnormally folded amyloid- $\beta(A \beta)$, tau proteins in extracellular amyloid plaques, and intracellular neurofibrillary tangles, which initiate oxidative stress, neuroinflammation, and lipid metabolism dysregulation, resulting in synaptic dysfunction, neurodegeneration, and neuron death in the brain $(3,4)$. Clinically, AD patients experience episodic memory deficits in the early stage of disease and gradually progress to cognitive impairment and declined independent self-care abilities for years (5). Despite the advancements in the development of novel biomarkers, imaging tools, therapeutic drugs, and patient care in the past decade, the prognosis of $A D$ patients remains dismal due to the lack of clarity about mechanisms underlying $A D$ pathology (2). Therefore, the elucidation of the mechanism underlying $A D$ development and progression might fuel the investigation of potential treatment targets for slowing the progression of $A D$ or even curing $A D$.

MicroRNAs (miRNAs) consist of a class of endogenous short non-coding RNA molecules with approximately 19 to 25 nucleotides in length, which are proposed as essential regulators for gene expression at the posttranscriptional level via targeting mRNA for degradation or translation inhibition $(6,7)$. miRNAs are reported to participate in the regulation of neuronal differentiation, dendrite spine morphology, and synaptic plasticity $(8,9)$. Among these identified miRNAs, microRNA (miR)-146a is

Correspondence: Dongmei Pan: <dongxianping175248@163.com> | Li Zhao: <licaorang2955@126.com>

${ }^{*}$ These authors contributed equally to this work as co-first authors. 
reported to be upregulated in brain regions targeted by $A D$ neuropathology such as the superior temporal lobe neocortex and hippocampus (10). Furthermore, one study showed that miR-146a is upregulated in the AD brain and mediates the modulation of complement factor $\mathrm{H}(\mathrm{CFH})$ that is involved in the inflammatory response regulation (11). Another study showed that miR-146a induces cell apoptosis by suppressing the low density lipoproteinrelated protein 2 (Lrp2)/Akt in SH-SY5Y cell lines (12). In addition, miR-146a/STAT1/MYC pathway is identified to be essential for regulating the progression of $A D$ by bioinformatics analysis (13). On these bases, we conducted this study with the aim to explore the effect of miR146 a inhibition on regulating cell apoptosis, total neurite outgrowth, inflammation, and STAT1/MYC pathway in cellular AD models.

\section{Material and Methods}

\section{Cell culture}

Rat pheochromocytoma (PC12) cell line was obtained from Cell Resource Center of Shanghai Institute of Life Sciences, Chinese Academy of Sciences (China) and cultured in DMEM medium (Gibco, USA) containing 5\% fetal bovine serum (FBS) (Gibco), 10\% horse serum (Gibco), $100 \mathrm{IU} / \mathrm{mL}$ penicillin, and $100 \mu \mathrm{g} / \mathrm{mL}$ streptomycin (Gibco). The PC12 cells were maintained at $37^{\circ} \mathrm{C}$ in a $95 \%$ humidified incubator with $5 \% \quad \mathrm{CO}_{2}$. Rat primary cortical neurons were isolated from the brain cortex of Sprague Dawley rat embryos on embryonic day 16 . The rat was purchased from Shanghai Lab Research Center (China), and the isolation and culture of rat embryo primary cortical neurons were performed according to the methods described in a previous study (14). The animal experiments were approved by the Institutional Animal Care and Use Committee of Huangshi Central Hospital and were conducted in line with the guidelines of the Care and Use of Laboratory Animals. Experiments were performed in biological triplicates, with only one technical replicate each.

\section{Cellular AD model construction}

For the PC12 cellular AD model construction, oligomerized $A \beta 1-42$ was prepared beforehand. $A \beta 1-42$ (Sigma, USA) was dissolved in dimethyl sulfoxide (DMSO) (Sigma) to a final concentration of $1 \mathrm{mM}$, sealed in an EP tube, and was incubated at $37^{\circ} \mathrm{C}$ for 7 days to promote aggregation. Following incubation, the $A \beta 1-42$ DMSO solution was diluted to $1 \mu \mathrm{M}$ in serum-free medium for subsequent use. Induction differentiation by nerve growth factor (NGF) was then performed. After discarding the initial medium, the PC12 cells were incubated in DMEM medium (Gibco) supplemented with $20 \mathrm{ng} / \mathrm{mL}$ NGF (Sigma) and $10 \%$ FBS (Gibco) for $72 \mathrm{~h}$ at $37^{\circ} \mathrm{C}$. Finally, NGF-stimulated PC12 cells were treated with $1 \mu \mathrm{M}$ oligomerized $A \beta 1-42$ peptides for $24 \mathrm{~h}$ to construct the cellular $A D$ model.

As for the cortical neuron cellular AD model construction, the rat embryo primary cortical neurons were also treated with $1 \mu \mathrm{M}$ oligomerized $\mathrm{A} \beta 1-42$ peptides for $24 \mathrm{~h}$. After cellular $A D$ model construction, using cells without A 1 1-42 treatment as control, the cell viability in PC12 and cortical neuron cellular $A D$ models was assessed using the Cell Counting kit-8 (CCK-8, Dojindo, Japan) in accordance with manufacturer's manual. miR-146a expressions in both cellular $A D$ models were determined by reverse transcription-quantitative polymerase chain reaction ( $R T-q P C R)$.

\section{Transfection}

The miR-146a inhibitor and negative-control inhibitor were purchased from Guangzhou RiboBio Co., LTD. (China). The PC12 and cortical neuron cellular AD models were transfected with miR-146a inhibitor and negativecontrol (NC) inhibitor, respectively, by Lipofectamine 2000 (Invitrogen, USA); the cells were termed as miR-inhibitor group and NC-inhibitor group, respectively. miR-146a expressions in both groups were detected by RT-qPCR after $24-\mathrm{h}$ incubation at $37^{\circ} \mathrm{C}$.

\section{Cell apoptosis detection}

At $48 \mathrm{~h}$ after transfection, cell apoptosis in both groups was detected by Hoechst/PI assay with the use of Hoechst 33342 (Sigma) and propidium iodide (PI) (Sigma). The procedures of Hoechst/PI assay were performed according to a previous study (15). Graphics were obtained using an inversion fluorescence microscope (Leica, Germany), and the cell apoptosis rate was calculated using the damaged cells divided by the total cells in the visual field.

\section{Total neurite outgrowth detection}

Cellular morphology and neurite outgrowth were observed with a microscope (Leica, Germany) at $48 \mathrm{~h}$ after transfection. The neurite outgrowth was quantified using Imaging software Presage (Advanced Imaging Concepts, Inc., USA), and the total neurite outgrowth per cell was calculated using total length of neurite outgrowth of cells divided by total cell counts in the visual field (15).

\section{Inflammatory cytokines detection}

Supernatant in both groups was collected at $48 \mathrm{~h}$ after transfection, and the inflammatory cytokines including tumor necrosis factor $\alpha$ (TNF- $\alpha$ ), interleukin (IL)-1 $\beta$, IL-6, and IL-17 in the supernatant were detected using commercial enzyme-linked immune-sorbent (ELISA) kits (SigmaAldrich, USA) following the manufacturer's manuals.

\section{Pathway detection}

According to a previous study, the miR-146a/STAT1/ MYC pathway may play a crucial role in $A D$ (13). 
Consequently, to validate whether the STAT1/MYC was regulated by miR-146a in $A D$ models, we detected the STAT1 and c-Myc expression in both groups using RT-qPCR and western blot assay at $48 \mathrm{~h}$ after transfection. Furthermore, to validate the hypothetical explanation (whether AKT/p-AKT was regulated by miR-146a in AD models), we detected the AKT and phosphorylated AKT ( $\mathrm{p}-\mathrm{AKT}$ ) protein expressions in both groups using western blot assay. The western blot assay was carried out according to the procedures described in a previous study (15). In brief, RIPA Buffer (Sigma) was used for protein extraction. Pierce ${ }^{\mathrm{TM}}$ BCA Protein Assay kit (Thermo Scientific, USA) was used for protein quantification, antiSTAT1 antibody (1:1000 dilution, Abcam, UK), anti-c-Myc antibody (1:1000 dilution, Abcam), anti-AKT antibody (1:500 dilution, Abcam), and anti-p-AKT antibody (1:500 dilution, Abcam) were used as the primary antibody, and goat anti-rabbit lgG H\&L (HRP) (1:10000 dilution, Abcam) was used as the secondary antibody. Novex ${ }^{\mathrm{TM}} \mathrm{ECL}$ Chemiluminescent Substrate Reagent kit (Invitrogen) was used for chemiluminescence. RT-qPCR was performed as described below.

\section{RT-qPCR}

Total RNA was obtained from the PC12 cells and rat primary cortical neurons by RNeasy Protect Mini kit (Qiagen, Germany), and then was reversely transcribed into complementary DNA (cDNA) by ReverTra Ace ${ }^{\mathbb{R}}$
qPCR RT Master mix (Toyobo, Japan). Subsequently, PCR amplification of CDNA was conducted by SYBR ${ }^{\circledR}$ Green Realtime PCR Master mix (Toyobo). The relative expressions of miR-146a, STAT1, and c-Myc were normalized by $2^{-\triangle \triangle \mathrm{Ct}}$ method; $U 6$ was used as an internal reference for miR-146a and GAPDH was used as an internal reference for both STAT1 and c-Myc. Of note, the same amount of RNA was used for the miR-146a detection in each group (control, $A \beta 1-42$ treated, NCinhibitor, and miR-inhibitor groups) to balance different cell number. The applied primer sequences were as follows: miR-146a (miR-146a-3p), F: 5'-ACACTC CAGCTGGGACCTGTGAAGTTCAGT-3', R: 5'-TGTCGT GGAGTCGGCAATTC-3'; U6, F: 5'-GCTTCGGCAGCA CATATACTA-3'; R: 5'-ATGGAACGCTTCACGAATTTGC3'; STAT1: F: 5'-TTGTGGAGTGGAAGCGAAGAC-3'; R: 5'-TGCTGGAAGAGGACGAAGGT-3'; c-Myc: F: 5'-GCTC TGCTCTCCGTCCTATGT-3', R: 5'-CAGTCCTGGATGAT GATGTTCTTGA-3'; GAPDH: F: 5'-GAGTCCACTGGCG TCTTCAC-3'; R: 5'-ATCTTGAGGCTGTTGTCATACTTC $\mathrm{T}-3^{\prime}$.

\section{Statistical analysis}

Data are reported as bar plots (means \pm SD) and analyzed by the GraphPad Prism 7.01 software (GraphPad Inc., USA). Comparison between two groups was determined by the unpaired $t$-test. $\mathrm{P}<0.05$ indicated statistical significance.
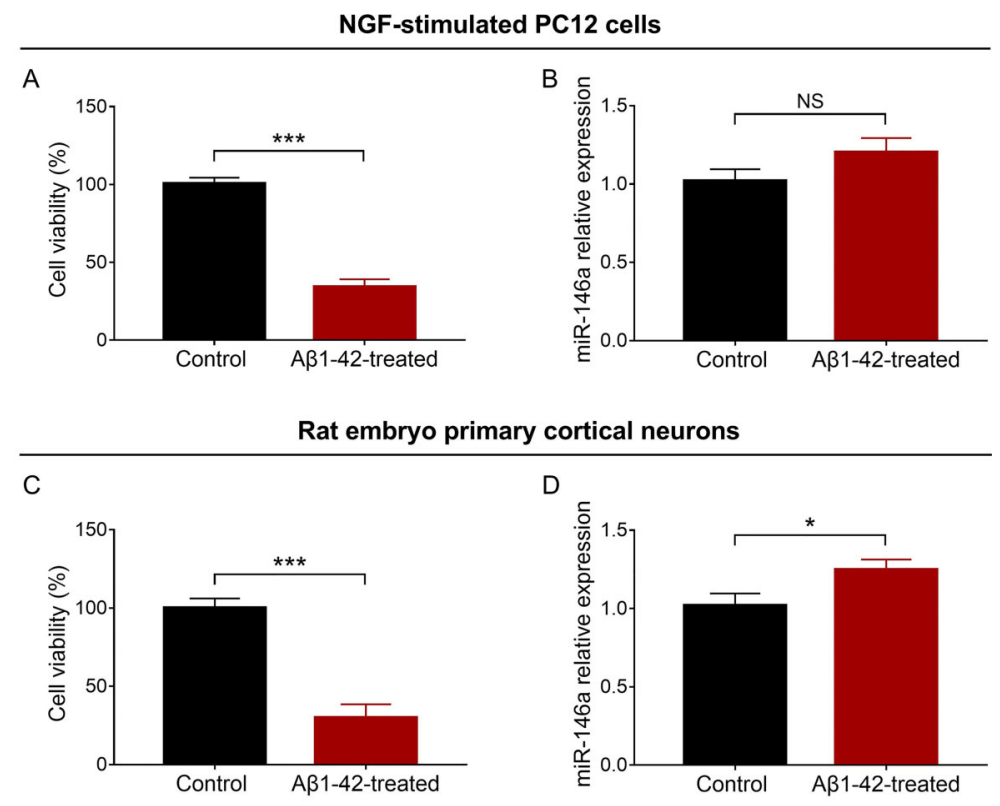

Figure 1. Comparison of cell viability $(\mathbf{A})$ and miR-146a relative expression (B) between control and $A \beta 1-42$-treated groups in the NGF-stimulated PC12 cellular AD model and in the rat embryo primary cortical neuron cellular AD model (C and D). miR: microRNA; AD: Alzheimer's disease; NGF: nerve growth factor; NS: not significant. Data are reported as means $\pm S D$. ${ }^{*} P<0.05,{ }^{* \star *} P<0.001$ $(t$-test). 


\section{Results}

Comparison of cell viability and $\mathrm{miR}-146 \mathrm{a}$ relative expression after $A \beta 1-42$ treatment

In NGF-stimulated PC12 cells, cell viability was decreased in the $A \beta 1-42$-treated group compared with the control group $(P<0.001)$ (Figure $1 A)$. No difference of miR-146a relative expression was observed between the $A \beta 1-42-t r e a t e d$ group and control group $(P>0.05)$ (Figure $1 \mathrm{~B})$. As for rat embryo primary cortical neurons, cell viability was attenuated in the $A \beta 1-42$-treated group compared with the control group $(P<0.001)$ (Figure $1 \mathrm{C})$ and miR-146a relative expression was increased in the A 1 1-42-treated group compared with the control group $(P<0.05)$ (Figure 1D).

\section{miR-146a relative expression and cell viability after} transfection

After transfection, miR-146a relative expression was greatly reduced in the miR-inhibitor group compared with the NC-inhibitor group in the PC12 cellular AD model $(P<0.001)$ (Figure $2 A)$. miR-146a relative expression was also significantly lower in the miR-inhibitor group than in the NC-inhibitor group in the cortical neuron cellular $A D$ model $(P<0.001)$ (Figure $2 B)$. Cell viability was not different between the miR-inhibitor group and the NCinhibitor group in both $\mathrm{PC} 12(\mathrm{P}>0.05)$ (Supplementary Figure S1A) and cortical neuron cellular $A D$ models $(P>0.05)$ (Supplementary Figure S1B).

\section{miR-146a inhibition reduced cell apoptosis}

In PC12 and cortical neuron cellular AD models, cell apoptosis was attenuated in the miR-inhibitor group compared with the NC-inhibitor group $(\mathrm{P}<0.05)$ (Figure 3A-D).

miR-146a inhibition promoted total neurite outgrowth

In PC12 and cortical neuron cellular AD models, total neurite outgrowth was increased in the miR-inhibitor group compared with the $\mathrm{NC}$-inhibitor group $(\mathrm{P}<0.01)$ (Figure 4A-D).

\section{miR-146a inhibition decreased inflammation}

In both PC12 and cortical neuron cellular AD models, supernatant TNF- $\alpha(P<0.01)$ (Figure $5 A$ and $E), I L-1 \beta$ $(P<0.001)$ (Figure 5B and F), IL-6 $(P<0.001)$ (Figure 5C and $G)$, and $I L-17(P<0.01)$ (Figure $5 D$ and $H$ ) levels were attenuated in the miR-inhibitor group compared with the NC-inhibitor.

miR-146a inhibition suppressed STAT1/MYC pathway In the PC12 cellular AD model, STAT1 $(P<0.001)$ and c-Myc $(P<0.01)$ mRNA and protein expressions were reduced in the miR-inhibitor group compared with the NCinhibitor group (Figure 6A, B, and C). In the cortical neuron cellular AD model, STAT1 $(P<0.01)$ and $c-M y c ~(P<0.01)$

\section{PC12 cellular AD model}

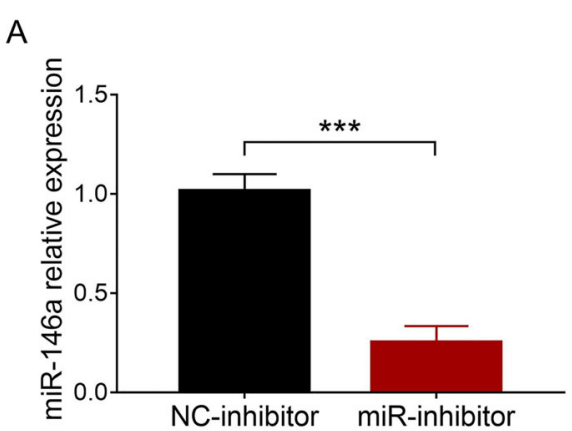

\section{Cortical neuron cellular AD model}

B

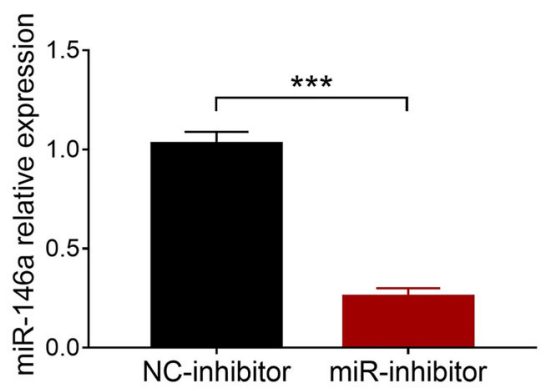

Figure 2. Comparison of miR-146a relative expression between NC-inhibitor group and miR-inhibitor group in PC12 (A) and cortical neuron $(B)$ cellular AD models. miR: microRNA; NC: negative control; $A D$ : Alzheimer's disease. Data are reported as means \pm SD. ${ }^{* * *} \mathrm{P}<0.001$ ( $t$-test).

mRNA and protein expressions were also lower in the miR-inhibitor group than in the NC-inhibitor group (Figure 6E, F, and G). In the PC12 cellular AD model, p-AKT protein expression was increased, while AKT protein expression did not differ in the miR-inhibitor group compared with the NC-inhibitor group (Figure 6D). In the cortical neuron cellular AD model, the findings were similar (Figure 6H).

\section{Discussion}

From the present study, we determined that miR-146a inhibition promoted total neurite outgrowth and suppressed cell apoptosis and inflammation as well as the STAT1/MYC pathway in both PC12 and cortical neuron cellular AD models.

$A D$, a complex age-related neurodegenerative disorder, is marked by two core pathological protein abnormalities, toxic $A \beta$ aggregation and hyperphosphorylated tau protein, inducing loss of connections between cells and extensive neurodegeneration over time (16). In addition to the well-studied $A \beta$ and tau pathology, lifestyle, vascular risk factors, and genetic susceptibility have been 


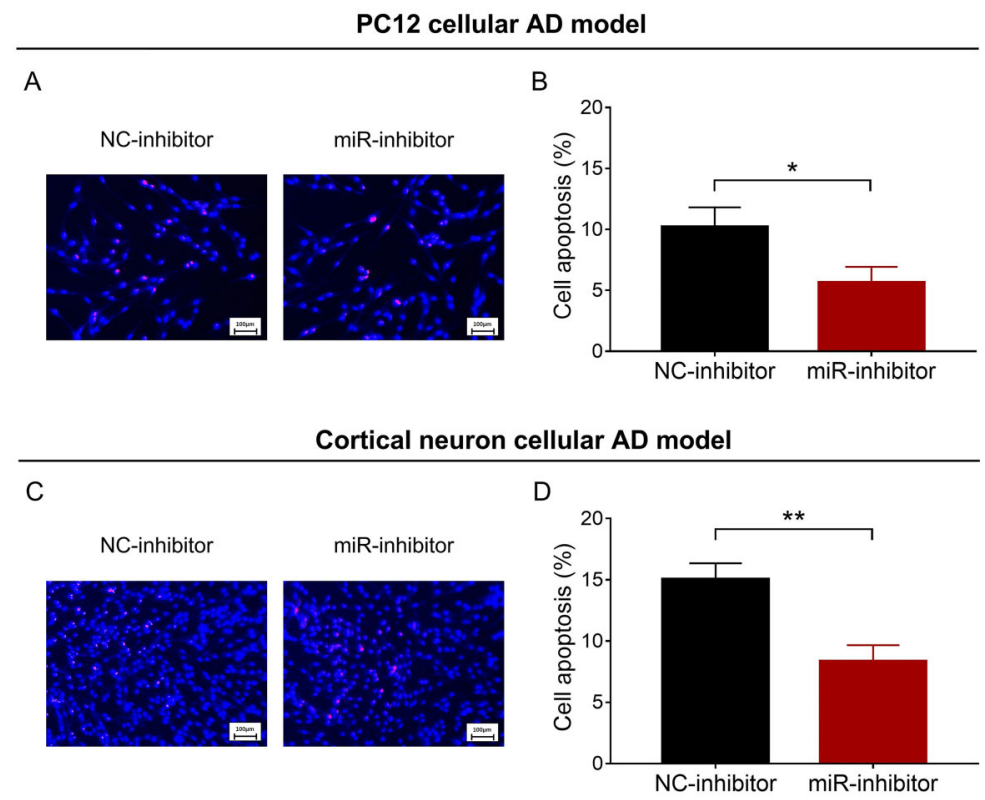

Figure 3. Cell apoptosis in NC-inhibitor group and miR-inhibitor group in PC12 (A and $\mathbf{B}$ ) and cortical neuron (C and $\mathbf{D})$ cellular $A D$ models. Blue color indicates Hoechst-stained cells and red color indicates PI-stained cells (scale bar: $100 \mu \mathrm{m}$ ). miR: microRNA; NC: negative control; AD: Alzheimer's disease; PI: propidium iodide. Data are reported as means $\pm \mathrm{SD}$. ${ }^{*} \mathrm{P}<0.05$, ${ }^{* *} \mathrm{P}<0.01$ ( $t$-test).

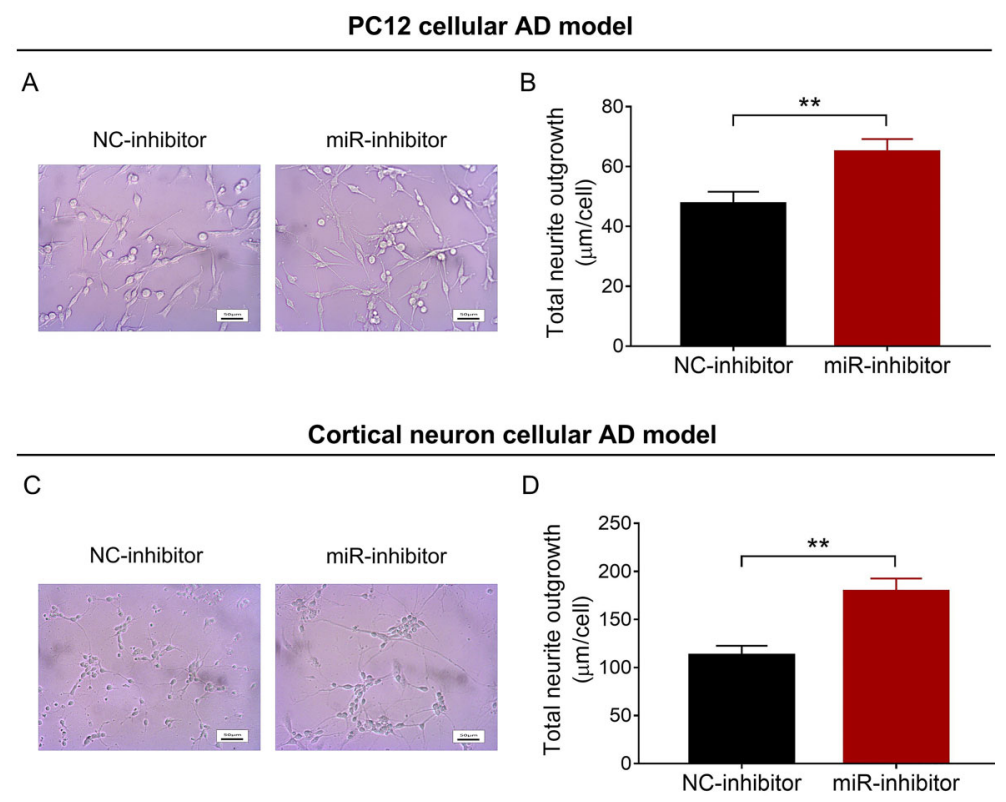

Figure 4. Total neurite outgrowth in NC-inhibitor group and miR-inhibitor group in PC12 (A and B) and cortical neuron (C and D) cellular AD models (scale bar: $50 \mu \mathrm{m}$ ). miR: microRNA; NC: negative control; $A D$ : Alzheimer's disease. Data are reported as means \pm SD. ${ }^{* \star} \mathrm{P}<0.01$ (t-test)

proposed as major contributors to the complex etiology of $A D(3)$. However, the genetic mechanisms underlying $A D$ are still poorly understood. Following the development and progress in the genome-wide association studies and next-generation sequencing in recent years, extensive research on microRNA has provided new insights into the pathogenesis of neurodegenerative diseases (9). It has been reported that many miRNAs express abundantly in 


\section{PC12 cellular AD model}

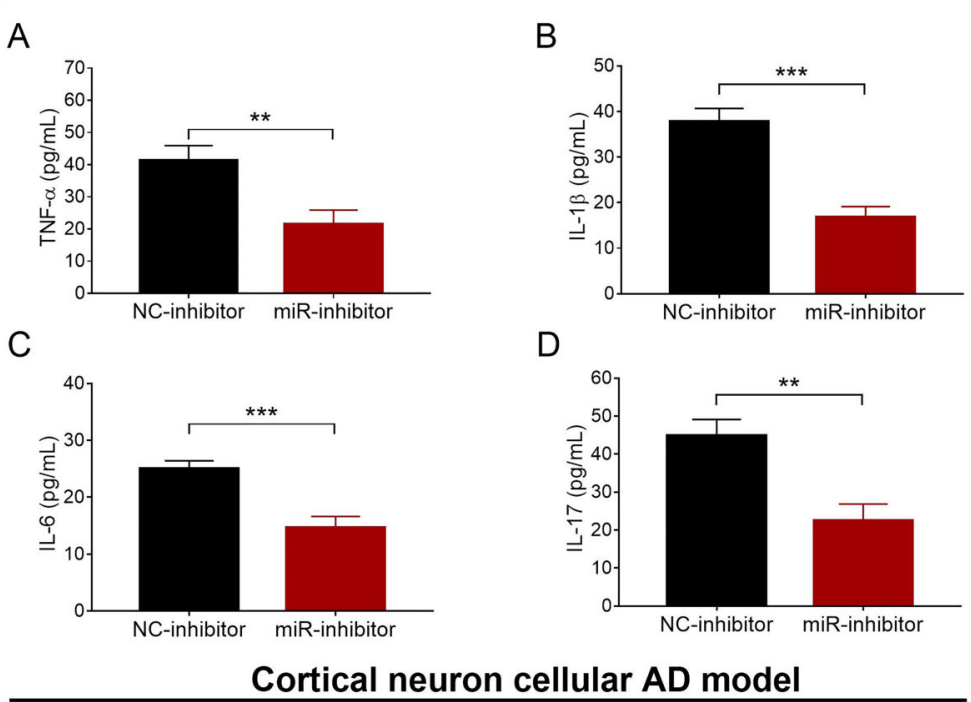

E

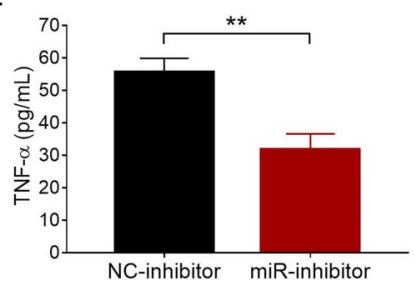

G

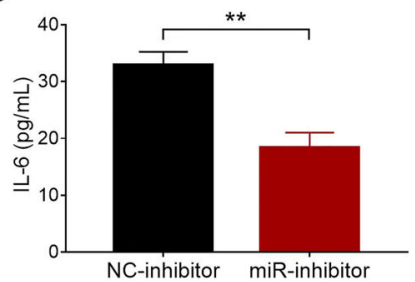

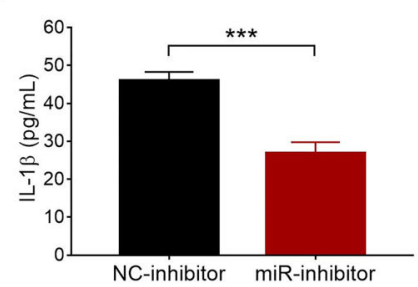

$\mathrm{H}$

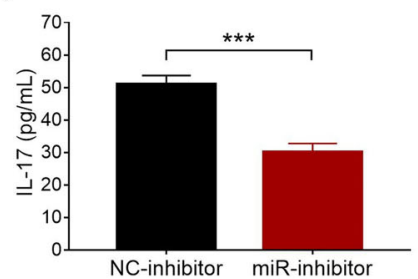

Figure 5. Comparison of supernatant TNF- $\alpha$, IL-1 $\beta$, IL-6, and IL-17 between NC-inhibitor group and miR-inhibitor group in PC12 (A-D) and cortical neuron (E-H) cellular AD models. miR: microRNA; TNF: tumor necrosis factor; IL: interleukin; NC: negative control; AD: Alzheimer's disease. Data are reported as means $\pm S D$. ${ }^{* \star} P<0.01,{ }^{* \star *} P<0.001$ (t-test).

the central nervous system and play a vital role in regulating neuron differentiation, synaptic plasticity, neurite outgrowth, and neurodegeneration $(8,9)$.

As one of the most extensively studied miRNAs, miR$146 a$ is proposed as a key regulator of the innate immune and inflammatory responses in certain immunological and brain cell types, which has been implicated in the pathogenesis of $A D(12,17,18)$. For instance, miR-146a triggers the downregulation of interleukin-1 receptorassociated kinase (IRAK)-1 and compensatory upregulation of IRAK-2, which drives intensified inflammatory responses in the hippocampus and neocortex of the $A D$ brain and stress human astroglial cells in primary culture (18). Another study, based on human neuroblastoma
SH-SY5Y cells, reveals that miR-146a inhibits Lrp2 protein expression and reduces subsequent Akt activation as well as pro-apoptotic caspases- 3 induction, which ultimately elevates cell apoptosis in $A D$ (12). In addition, miR-146a is associated with senile plaque density and synaptic pathology in Tg2576 and in 5xFAD transgenic mouse models (17).

Our results were in line with this aforementioned evidence, which showed that cell apoptosis and inflammation (reflected by supernatant TNF- $\alpha$, IL-1 $1 \beta$, IL- 6 , and IL-17 levels) were decreased in miR-146a-inhibitor-treated cells compared with NC inhibitor-treated cells in both PC12 and cortical neuron cellular AD models. Herein, we proposed several explanations. First, miR-146a inhibition 


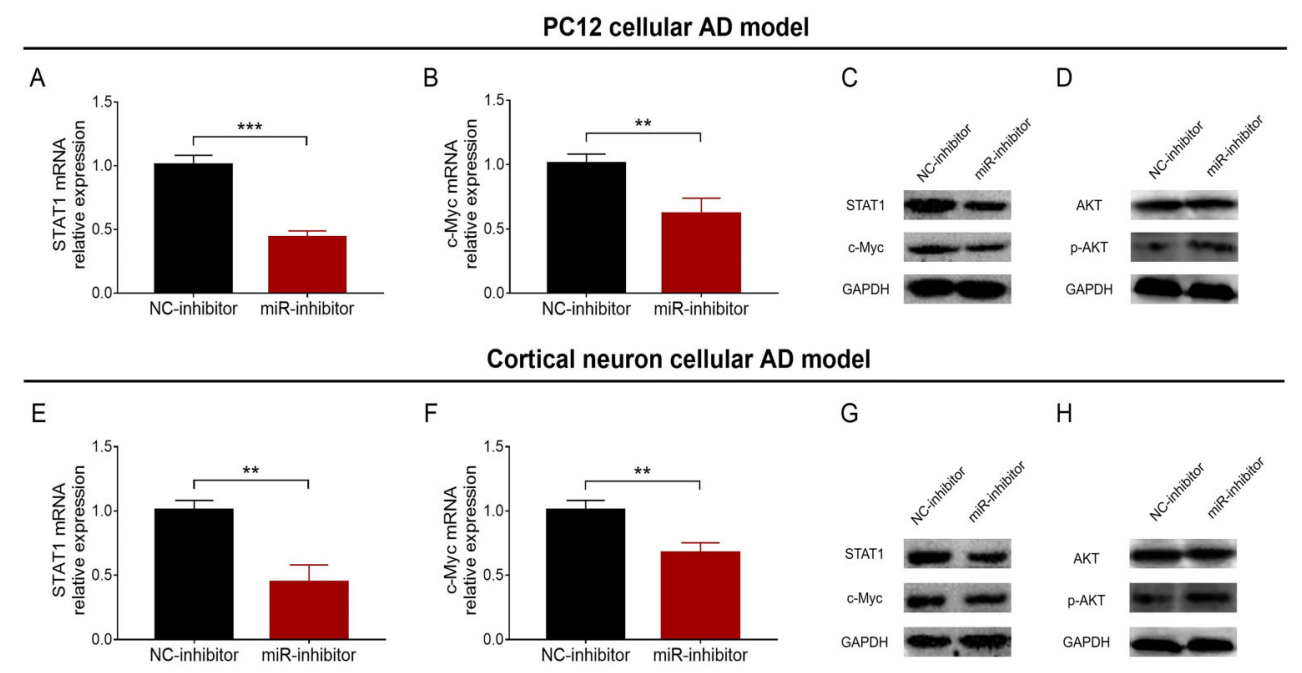

Figure 6. Comparison of STAT1 mRNA (A) and c-Myc mRNA (B) relative expressions and STAT1 and c-Myc protein expressions (C and D) between NC-inhibitor group and miR-inhibitor group in PC12 cellular AD model. Comparison of STAT1 mRNA (E) and c-Myc mRNA (F) relative expressions and STAT1 and c-Myc protein expressions $(\mathbf{G}$ and $\mathbf{H})$ between NC-inhibitor group and miR-inhibitor group in cortical neuron cellular AD model. miR: microRNA; STAT1: signal transducer and activator of transcription 1; NC: negative control; mRNA: messenger RNA; AD: Alzheimer's disease; $p-A K T$ : phosphorylated-AKT. Data are reported as means $\pm S D$. ${ }^{* *} \mathrm{P}<0.01$, ${ }^{* * *} \mathrm{P}<0.001$ (t-test).

might have enhanced the translation of downstream proteins (e.g., low-density lipoprotein receptor-related protein-2), which mediated the activation of AKT, the induction of pro-apoptotic caspase-3, and suppressed cell apoptosis in both AD models (12). Meanwhile, miR146a inhibition increased p-AKT protein expression, and it did not change AKT protein expression in either cellular AD model, which supported our hypothetical explanation that miR-146a inhibition might induce the activation of AKT in cellular AD models. Second, miR-146a inhibition might have facilitated specific inflammation-relevant mRNAs such as $\mathrm{CFH}$, interleukin-1 receptor-associated kinase 1, and tetraspanin-12, which inhibited the release of pro-inflammatory cytokines and decreased sustained inflammation in both $A D$ models extensively $(11,18)$.

Furthermore, we also observed that total neurite outgrowth was elevated in the miR-146a inhibitor-treated cells compared with the NC inhibitor-treated cells in both cellular $A D$ models. The possible reason could be that miR-146a inhibition might have upregulated the expression of certain brain-essential mRNA targets (e.g., amyloid precursor protein), which increased the neurite outgrowth in the cellular AD models $(19,20)$. However, this speculation needs further validation.

In a previous study, consistent differentially expressed genes (cDEGs) were obtained by a meta-analysis of multiple gene microarray datasets, and differentially expressed miRNA (DEmiRs) were retrieved from one miRNA expression profile (GSE16759). Then, the potential active transcription factor (TF)-miRNA regulatory subnetwork in $A D$ was obtained by mapping the cDEGs and DEmiRs to the curated TF-miRNA regulatory network (constructed by integrating TRANSFAC, TransmiR, miRTarBase, miRecords and TarBase data resources) as active seed nodes and connecting active seed nodes with their immediate neighbors. Next, potential active TFmiRNA pathways in $A D$ were found by breadth-firstsearch method. Lastly, according to the known AD-related genes and miRNAs (derived from the GeneCards database), nine active TF-miRNA pathways in AD were identified by hypergeometric test, among which miR$146 \mathrm{a} / \mathrm{STAT} 1 / \mathrm{MYC}$ is the source of all nine pathways and may play a critical role in the progression of $A D(13)$. Thus, the present study evaluated the effect of miR-146a inhibition on regulating STAT1/MYC pathway in AD. We found that STAT1 and c-Myc mRNA and protein expressions were decreased in the miR-146a inhibitor-treated cells compared with NC inhibitor treated cells in both cellular AD models, which implied that the miR-146a inhibition suppressed the STAT1/MYC pathway in AD. In addition, it might also explain the effect of miR-146a inhibition on suppressing cell apoptosis and inflammation and enhancing total neurite outgrowth in both $A D$ models as follows: STAT1 is known to induce inflammation by increasing the expression of pro-inflammatory mediators and to mediate cell apoptosis by triggering the transcriptional activation of death-modulating genes (e.g., caspases, death receptors, and ligands) $(21,22)$. Meanwhile, MYC might induce neurodegeneration via activating the cell cycle in the neurons (23). Additionally, suppression of STAT1 was reported to be accompanied by decreased 
MYC expression (22). Combining the aforementioned evidence and the findings of our cellular experiments, miR-146a inhibition might attenuate cell apoptosis and inflammation and elevate total neurite outgrowth through suppressing the STAT1/MYC pathway. However, our speculation requires further verification. Due to the limited budget, only cellular AD models were used in the investigations, thus, further animal study is needed to validate our results.

\section{References}

1. Dourlen $P$, Kilinc D, Malmanche N, Chapuis J,Lambert JC. The new genetic landscape of Alzheimer's disease: from amyloid cascade to genetically driven synaptic failure hypothesis? Acta Neuropathol 2019; 138: 221-236, doi: 10.1007/ s00401-019-02004-0.

2. Cao J, Hou J, Ping J, Cai D. Advances in developing novel therapeutic strategies for Alzheimer's disease. Mol Neurodegener 2018; 13: 64, doi: 10.1186/s13024-018-0299-8.

3. Scheltens P, Blennow K, Breteler MM, de Strooper B, Frisoni GB, Salloway S, et al. Alzheimer's disease. Lancet 2016; 388: 505-517, doi: 10.1016/S0140-6736(15)01124-1.

4. Canter RG, Penney J, Tsai LH. The road to restoring neural circuits for the treatment of Alzheimer's disease. Nature 2016; 539: 187-196, doi: 10.1038/nature20412.

5. Weintraub S, Wicklund AH, Salmon DP. The neuropsychological profile of Alzheimer disease. Cold Spring Harb Perspect Med 2012; 2: a006171, doi: 10.1101/cshperspect. a006171.

6. Lu TX, Rothenberg ME. MicroRNA. J Allergy Clin Immunol 2018; 141: 1202-1207, doi: 10.1016/j.jaci.2017.08.034.

7. Bartel DP. MicroRNAs: genomics, biogenesis, mechanism, and function. Cell 2004; 116: 281-297, doi: 10.1016/S00928674(04)00045-5.

8. Femminella GD, Ferrara N, Rengo G. The emerging role of microRNAs in Alzheimer's disease. Front Physiol 2015; 6: 40, doi: $10.3389 /$ fphys.2015.00040.

9. Qiu L, Tan EK, Zeng L. MicroRNAs and neurodegenerative diseases. Adv Exp Med Biol 2015; 888: 85-105, doi: 10.1007/978-3-319-22671-2_6.

10. Sethi $P$, Lukiw WJ. Micro-RNA abundance and stability in human brain: specific alterations in Alzheimer's disease temporal lobe neocortex. Neurosci Lett 2009; 459: 100-104, doi: 10.1016/j.neulet.2009.04.052.

11. Lukiw WJ, Zhao Y, Cui JG. An NF-kappaB-sensitive micro RNA-146a-mediated inflammatory circuit in Alzheimer disease and in stressed human brain cells. $J$ Biol Chem 2008; 283: 31315-31322, doi: 10.1074/jbc.M805371200.

12. Zhang $B$, Wang LL, Ren RJ, Dammer EB, Zhang YF, Huang $Y$, et al. MicroRNA-146a represses LRP2 translation and leads to cell apoptosis in Alzheimer's disease. FEBS Lett 2016; 590: 2190-2200, doi: 10.1002/18733468.12229.

13. Jiang W, Zhang $Y$, Meng F, Lian B, Chen X, Yu X, et al. Identification of active transcription factor and miRNA regulatory pathways in Alzheimer's disease. Bioinformatics 2013; 29: 2596-2602, doi: 10.1093/bioinformatics/btt423.
In conclusion, miR-146a inhibition promoted total neurite outgrowth and suppressed cell apoptosis, inflammation, as well as STAT1/MYC pathway in PC12 and cortical neuron cellular $A D$ models, which could indicate miR-146a as a potential therapeutic target for AD.

\section{Supplementary Material}

Click to view [pdf].

14. Fang $M$, Wang J, Zhang $X$, Geng $Y$, Hu Z, Rudd JA, et al. The miR-124 regulates the expression of BACE1/betasecretase correlated with cell death in Alzheimer's disease. Toxicol Lett 2012; 209: 94-105, doi: 10.1016/j.toxlet.2011. 11.032.

15. Yang $H$, Wang $H$, Shu $Y$, Li X. miR-103 promotes neurite outgrowth and suppresses cells apoptosis by targeting prostaglandin-endoperoxide synthase 2 in cellular models of Alzheimer's disease. Front Cell Neurosci 2018; 12: 91, doi: 10.3389/fncel.2018.00091.

16. Kharma B, Baba T, Matsumura N, Kang HS, Hamanishi J, Murakami R, et al. STAT1 drives tumor progression in serous papillary endometrial cancer. Cancer Res 2014; 74: 6519-6530, doi: 10.1158/0008-5472.CAN-14-0847.

17. Li YY, Cui JG, Hill JM, Bhattacharjee S, Zhao Y, Lukiw WJ. Increased expression of miRNA-146a in Alzheimer's disease transgenic mouse models. Neurosci Lett 2011; 487: 94-98, doi: 10.1016/j.neulet.2010.09.079.

18. Cui JG, Li YY, Zhao Y, Bhattacharjee S, Lukiw WJ. Differential regulation of interleukin-1 receptor-associated kinase-1 (IRAK-1) and IRAK-2 by microRNA-146a and NFkappaB in stressed human astroglial cells and in Alzheimer disease. J Biol Chem 2010; 285: 38951-38960, doi: 10.1074/jbc.M110.178848.

19. Small DH, Clarris HL, Williamson TG, Reed G, Key B, Mok $\mathrm{SS}$, et al. Neurite-outgrowth regulating functions of the amyloid protein precursor of Alzheimer's disease. J Alzheimers Dis 1999; 1: 275-285, doi: 10.3233/JAD-1999-14-508.

20. Li K, Zhang J, Ji C, Wang L. MiR-144-3p and Its target gene beta-amyloid precursor protein regulate 1-Methyl-4-Phenyl1,2-3,6-Tetrahydropyridine-induced mitochondrial dysfunction. Mol Cells 2016; 39: 543-549, doi: 10.14348/molcells. 2016.0050.

21. Kim HS, Lee MS. STAT1 as a key modulator of cell death. Cell Signal 2007; 19: 454-465, doi: 10.1016/j.cellsig.2006. 09.003.

22. Sikorski K, Czerwoniec A, Bujnicki JM, Wesoly J, Bluyssen HA. STAT1 as a novel therapeutical target in pro-atherogenic signal integration of IFNgamma, TLR4 and IL-6 in vascular disease. Cytokine Growth Factor Rev 2011; 22: 211-219, doi: 10.1016/j.cytogfr.2011.06.003.

23. Lee HG, Casadesus G, Nunomura A, Zhu X, Castellani RJ, Richardson SL, et al. The neuronal expression of MYC causes a neurodegenerative phenotype in a novel transgenic mouse. Am J Pathol 2009; 174: 891-897, doi: 10.2353/ ajpath.2009.080583. 